\title{
The fabrication of color-tunable organic light-emitting diode displays via solution processing
}

\begin{abstract}
Fei Guo ${ }^{1,2}$, Andre Karl ${ }^{1}$, Qi-Fan Xue ${ }^{3}$, Kai Cheong Tam ${ }^{4}$, Karen Forberich ${ }^{1}$ and Christoph J Brabec ${ }^{1,4}$
Electroluminescent devices based on organic semiconductors have attracted significant attention owing to their promising applications in flat-panel displays. The conventional display pixel consisting of side-by-side arrayed red, green and blue subpixels represents the mature technology but bears an intrinsic deficiency of a low pixel density. Constructing an individual color-tunable pixel that comprises vertically stacked subpixels is considered an advanced technology. Although color-tunable organic light-emitting diodes (OLEDs) have been fabricated using the vacuum deposition of small molecules, the solution processing of conjugated polymers would enable a much simpler and inexpensive manufacturing process. Here we present the all-solution processing of color-tunable OLEDs comprising two vertically stacked polymer emitters. A thin layer of highly conducting and transparent silver nanowires is introduced as the intermediate charge injection contact, which allows the emission spectrum and intensity of the tandem devices to be seamlessly manipulated. To demonstrate a viable application of this technology, a 4-by-4 pixelated matrix color-tunable display was fabricated.
\end{abstract}

Light: Science \& Applications (2017) 6, e17094; doi:10.1038/lsa.2017.94; published online 17 November 2017

Keywords: color tunable; OLED display; organic light-emitting diode; silver nanowires; solution processing; tandem

\section{INTRODUCTION}

Organic light-emitting diodes (OLEDs) based on small organic molecules or conjugated polymers exhibit unique features of high brittleness, a low turn-on voltage and a wide range of colors ${ }^{1-3}$. In combination with their solution-processing compatibility, these favorable characteristics make OLED devices potential candidates for use in the manufacture of low-cost full-color displays ${ }^{4-6}$. Recently, thermally deposited OLEDs made of small molecules have found market applications in consumer electronic devices such as Samsung's and LG's flat-panel TVs and the Apple watch. Currently, the most successful full-color flat-panel display technology is based on a 'sideby-side' geometry, where the individual emitting pixel consists of three laterally arrayed red (R), green $(G)$ and blue (B) subpixels ${ }^{5-7}$. However, a major drawback of this 'side-by-side' geometry is the inherently restricted resolution caused by the low geometric fill factor.

It is known that many organic light-emitting materials exhibit large Franck-Condon red shifts between their absorption and emission spectra $^{8-10}$. A high electroluminescence performance can be achieved by using very thin films. These unique properties enable the fabrication of visibly semitransparent OLED devices ${ }^{11,12}$. The semitransparency of these organic thin films permits the manufacture of vertically stacked pixels by sequentially depositing two or more subdiodes on top of each other. Color-tunable emitting pixels can be realized by such a vertically stacked approach. Compared to the conventional side-by-side geometry, the vertically stacked architecture offers the advantages of easier device manufacture and a smaller pixel size and, thus, a higher fill factor ${ }^{13}$.

The essential task for the fabrication of vertically stacked colortunable OLED devices is to deposit a thin layer of a transparent conducting film as a common charge injection contact for the subdiodes. In the 1990s, Forrest and co-authors ${ }^{14,15}$ first conceived and fabricated color-tunable OLED devices by monolithically depositing small molecular diodes that were interconnected with thermally deposited metal alloys. Following these pioneering works, several groups fabricated color-tunable OLED cells via the vacuum deposition of small molecular emitting materials and analogous interconnection layers consisting of thin metal films (for example, $\mathrm{Au}$ and $\mathrm{Ag})^{16-19}$. However, all of the emitting materials and the intermediate layers in these devices were deposited via a high-vacuum process, which increased the complexity of the device fabrication. Moreover, the intermediate electrode made of thin metal films can absorb a significant amount of emitted light and introduce unwanted microcavity effects ${ }^{15,20}$. In contrast, the use of a solution process for the conjugated polymer emitters and the interface materials can result in a much simpler and less expensive fabrication process. Nevertheless, due to the challenge of introducing a highly transparent and conducting contact layer that can be solution-deposited between subdiodes, the realization of this technology in a solution-processing manner has lagged far behind its evaporation counterpart.

\footnotetext{
${ }^{1}$ Institute of Materials for Electronics and Energy Technology (i-MEET), Friedrich-Alexander University Erlangen-Nürnberg, Erlangen 91058 , Germany; ${ }^{2}$ Institute of New Energy Technology, College of Information and Technology, Jinan University, Guangzhou 510632, China; ${ }^{3}$ Institute of Polymer Optoelectronic Materials and Devices, State Key Laboratory of Luminescent Materials and Devices, South China University of Technology, Guangzhou 510640, China and ${ }^{4}$ Bavarian Center for Applied Energy Research (ZAE Bayern), Erlangen 91058, Germany

Correspondence: F Guo, Email: feigu086@gmail.com; KC Tam, Email: Eric.Tam@zae-bayern.de; CJ Brabec, Email: christoph.brabec@fau.de

Received 29 January 2017; revised 25 May 2017; accepted 28 May 2017; accepted article preview online 1 June 2017
} 
Two-terminal color-tunable OLED devices with a heterojunction configuration of $\mathrm{n}-\mathrm{i}-\mathrm{p}-\mathrm{i}-\mathrm{n}$ have been demonstrated as an alternative $^{21,22}$. In such a device, the middle p-type interlayer serves as a charge generation layer that generates holes for the two subdiodes. This approach was first demonstrated by Zhao et al in 2013 by thermal evaporation, and Park's group later utilized a solution processing method $^{21,22}$. The advantage of such a configuration is its simpler device processing, as the intermediate charge injection contact is not a prerequisite. However, due to the absence of the intermediate contacts, this geometry is not applicable for the construction of three-junction full-color-tunable devices.

In this article, we report for the first time three-terminal colortunable OLED devices based on sequential layer-by-layer deposition. In contrast to the earlier works that used vacuum-deposited metal films as the intermediate charge injection layer, we chose a highly transparent silver nanowire (AgNW) network for our color-tunable OLED devices. Through careful material selection in combination with rational interface engineering, we successfully implemented AgNWs between two LEDs by using only solution processing without significantly impairing the diode performance. The accessibility of the middle contact allows us to seamlessly manipulate the individual subdiodes, thereby achieving mixed colors between the primary colors with adjustable intensities. To illustrate a practical application of this technology, we have manufactured a matrix display that consists of 4-by-4 arrayed pixels.

\section{MATERIALS AND METHODS}

\section{Materials}

ITO-coated glass substrates with a sheet resistance of $15 \Omega \mathrm{sq}^{-1}$ and transmittance of $81 \%$ at $550 \mathrm{~nm}$ were received from Weidner Glas. The red-emitter (Livilux, SPR-001) and green-emitter (Livilux, SPG$-01 \mathrm{~T})$ were purchased from Merck (Darmstadt, Germany), and the blue-emitter PFO was purchased from Sigma-Aldrich (Darmstadt, Germany). Standard PEDOT:PSS (Clevios, P VP AI 4083) and neutral PEDOT:PSS (NT5-3417286/2) were obtained from Heraeus and Agfa, respectively. $\mathrm{ZnO}$ nanoparticle ink $(\mathrm{N}-10)$ and a $\mathrm{AgNW}$ dispersion were kindly supplied by Nanograde AG (Zurich, Switzerland) and Cambrios Technologies Corporation (Sunnyvale, CA, USA), respectively. All materials were used as received without further purification.

\section{Fabrication of color-tunable tandem devices}

Prior to the device fabrication, the ITO substrates were subjected to laser patterning and subsequently cleaned by ultra-sonication in acetone and isopropanol for $10 \mathrm{~min}$ each. In our color-tunable tandem OLED cells, all of the solution-processed layers were deposited using a doctor blade in an ambient atmosphere. Taking the material combination of green and red emitters as an example, on the cleaned ITO substrates, PEDOT:PSS (P VP Al 4083, 1:3 vol.\% diluted in isopropanol) was bladed and baked at $140{ }^{\circ} \mathrm{C}$ for $5 \mathrm{~min}$, and the front green emitter $\left(6 \mathrm{mg} \mathrm{ml}^{-1}\right.$ in toluene) was deposited on top of the dried PEDOT:PSS at $50{ }^{\circ} \mathrm{C}$. The film thickness of the layer was determined to be $\sim 80 \mathrm{~nm}$. The intermediate layers consisting of $\mathrm{ZnO}$, neutral PEDOT:PSS (N-PEDOT) and AgNWs were sequentially bladed at $50^{\circ} \mathrm{C}$ and annealed at $80^{\circ} \mathrm{C}$ for $5 \mathrm{~min}$ in air. The thicknesses of the three layers were $40 \mathrm{~nm}(\mathrm{ZnO}), 35 \mathrm{~nm}(\mathrm{~N}-\mathrm{PEDOT})$ and $100 \mathrm{~nm}$ (AgNWs), and the AgNW film showed a sheet resistance of $\sim 15 \Omega \mathrm{sq}^{-1}$. To prepare the top LED, PEDOT:PSS was first deposited on top of the AgNWs and baked at $120^{\circ} \mathrm{C}$ for 5 min. The back red emitter with a thickness of $\sim 80 \mathrm{~nm}$ was successively coated on top of the PEDOT:PSS layer at $55^{\circ} \mathrm{C}$. After all of the solution-processed layers were completed, Q-tips dipped in toluene were used to clean the edges of the substrate to expose the bottom ITO and middle AgNW contacts. To complete the device fabrication, a 10-nm thick Ca layer and a 100-nm-thick Ag layer were thermally evaporated on top of the red-emitting layer through a shadow mask. The emitting area of the device was determined by the intersection of three charge injection contacts. A similar procedure was used for the construction of redblue and green-blue tandem OLED devices.

\section{Color-tunable display fabrication}

The color-tunable display was fabricated by using a procedure similar to that used to prepare the tandem devices described above. To achieve individual color-tunable pixels, the bottom, middle and top charge-injecting contacts need to be fabricated in a stripe shape. In our passive matrix display demonstrations, the bottom ITO was patterned with a femtosecond laser. Upon the deposition of the bottom and top subdiodes, the middle and top AgNW stripe shape electrodes were prepared using a modified doctor blade. A detailed description of the fabrication process is presented in the section 'Manufacture of colortunable display' and the Supplementary Information.

\section{Characterization}

The transmittance spectra of the intermediate layers and semitransparent devices were recorded using a UV-vis-NIR spectrometer (Lambda 950, Perkin Elmer, Akron, OH, USA). The current density-voltage-luminance ( $\mathrm{J}-\mathrm{V}-\mathrm{L})$ characteristics were detected using a source measurement unit from BoTest by shifting the applied voltage from 0 to $8 \mathrm{~V}$ in $50 \mathrm{mV}$ increments. The luminance of the BoTest detector was calibrated using a CS200 spectroradiometer (Konica Minolta, Marunouchi, Japan). The film thicknesses of the layers were measured with a profilometer (Tencor Alpha Step D 100, Milpitas, CA, USA), and the EL spectra of the OLED devices were recorded using a home-built setup. The atomic force microscopy (AFM) and cross-sectional scanning electron microscopy (SEM) images were recorded with a NT-MDT Solver Nano (tapping mode) and JEOL JSM-7610F with an IB-19500CP cross-section polisher, respectively.

\section{RESULTS AND DISCUSSION}

\section{Single-junction OLEDs}

Three conjugated polymers emitting the primary colors of red, green and blue are used as the light-emitting materials for the construction of the color-tunable OLED devices. For reference, we first fabricate and evaluate the electroluminescence performance of the three lightemitting devices in their single-junction configurations. As shown in Figure $1 \mathrm{a}$, in these single-junction devices, solution-coated poly $(3,4$ ethylenedioxythiophene) polystyrene sulfonate (PEDOT:PSS) and thermally deposited $\mathrm{Ca}$ were used as the hole and electron injection layers, respectively. A detailed description of the device fabrication is presented in the Materials and methods. We note that a normal device architecture is chosen for our OLED cells primarily owing to its capability of replacing the air-sensitive electron injection layers (that is, $\mathrm{Ca}$ and $\mathrm{Ba}$ ) with solution-processed low work-function metal oxides (for example, $\mathrm{ZnO}$ and $\mathrm{TiO}_{2}$ ). It is discussed below that the incorporation of these nonreactive metal oxides on top of the emitting layers further enables the deposition of several upper layers by solution processing.

Figure $1 \mathrm{~b}$ shows the electroluminescent spectra of the three RGB OLED devices recorded in their reference devices. The emission peaks of the red-, green- and blue-emitting diodes are located at 650, 514 and $433 \mathrm{~nm}$, respectively. The CIE 1931 color space chromaticity coordinates are calculated to be $(0.67,0.33)$ for the red, $(0.33,0.59)$ for the green and $(0.18,0.18)$ for the blue OLED devices, suggesting 
a

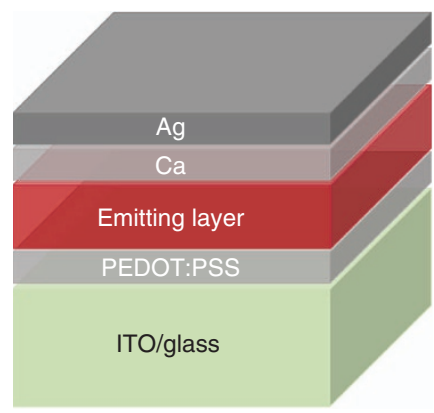

C

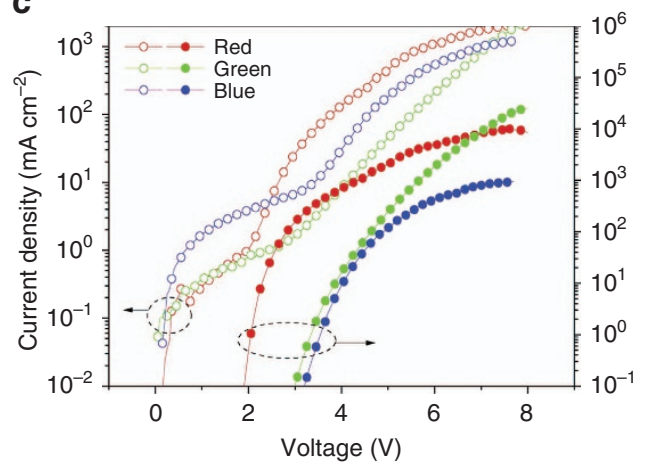

b
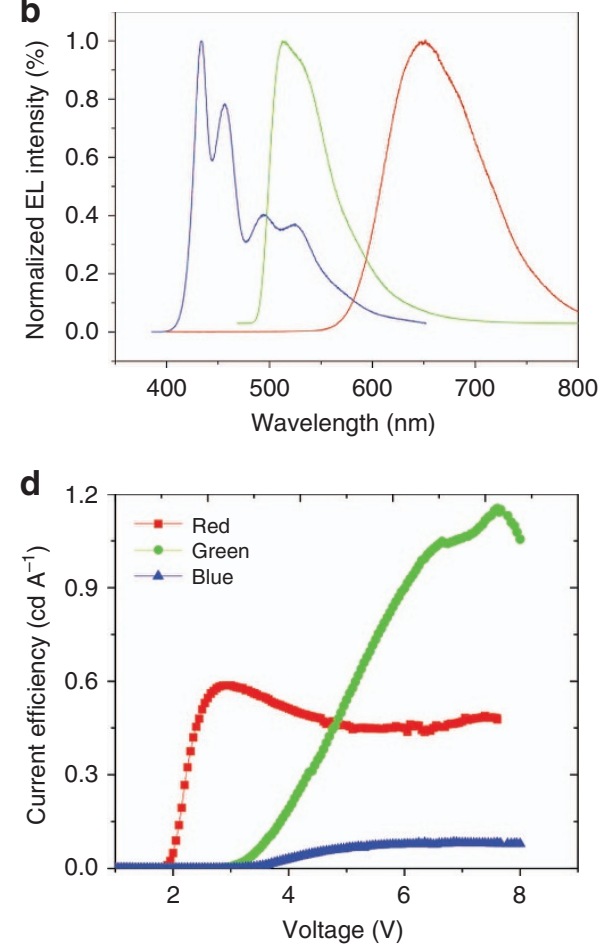

Figure 1 Device structure and electroluminescence performance of the three single-junction OLED devices with red-, green- and blue-emitting polymers. (a) Schematic architecture of the single-junction reference cell with a device structure of 'ITO/PEDOT:PSS/Emitting layer/Ca/Ag'. (b) Electroluminescent spectra of the red, green and blue OLED devices. (c) Current density (open circle) and luminance (solid circle) as functions of the voltage characteristics of the three single-junction reference diodes. (d) Current efficiency of the three reference devices.

fairly pure primary colors (Supplementary Fig. 1). The J-V-L characteristics of the three OLED cells are displayed in Figure 1c. It can be seen that the cells turn on at 1.95, 3.0 and $3.2 \mathrm{~V}$ (defined at $0.1 \mathrm{~cd} \mathrm{~m}^{-2}$ ) and that the maxima brightness reaches 9802, 25232 and $963 \mathrm{~cd} \mathrm{~m}^{-2}$ at $8 \mathrm{~V}$ for the red, green and blue diodes, respectively. Figure 1d shows the current efficiencies of the three reference devices. Compared to thermally deposited small-molecular OLED devices, our devices give low peak efficiencies of $0.58,1.15$ and $0.9 \mathrm{~cd} \mathrm{~A}^{-1}$ for the red, green and blue diodes, respectively. These relatively low efficiencies are probably due to the absence of electron- and hole-blocking layers, which are commonly used to enhance the charge recombination in small-molecular OLEDs.

\section{Interface engineering for AgNW-based OLED devices}

Having noted that the most important step in the successful demonstration of color-tunable tandem OLED cells is to incorporate a transparent and conducting interlayer as a common charge injection contact for the subdiodes, it is worth noting that solution-processed charge generation layers consisting of n-type and p-type semiconductors have been reported by several groups to fabricate white light tandem OLED devices ${ }^{23-27}$. In contrast, the preparation of a transparent and conducting intermediate contact for manufacturing colortunable tandem OLED cells has been realized by solely vacuum deposition ${ }^{16-19}$. There are indeed several challenges in constructing an efficient and robust intermediate layer fully by solution processing. First, the intermediate conducting layer should possess a low sheet resistance to minimize electrical losses. Second, the intermediate layers should be highly transparent to efficiently transmit the photons emitted from the back diode. Third, from the processing point of view, the intermediate layer should be sufficiently robust to withstand the processing solvents of the upper layers that can cause dissolution. Fourth, to facilitate carrier injection, low ohmic contacts should be formed between these intermediate layers and the active layer. Finally, the intermediate layers should be relatively smooth to avoid shortcircuiting the subdiodes.

Through a combination of rational material selection and interface engineering, an efficient intermediate layer is developed to effectively addresses these challenges. We first choose silver nanowires (AgNWs) as the charge injection contact for our color-tunable tandem devices, primarily because of their outstanding optoelectronic properties and facile solution processability ${ }^{28-31}$. Supplementary Fig. 2a shows the SEM image and transmittance spectrum of the AgNW film deposited from isopropanol-based solution. The high length-to-diameter aspect ratio, which can reach 1000 (diameter of $30 \mu \mathrm{m}$ and $30 \mathrm{~nm}$ in length), of the individual nanowires result in a low sheet resistance of $20 \Omega \mathrm{sq}^{-1}$ and high transparency of $86 \%$ (at $550 \mathrm{~nm}$ ) for a $150-\mathrm{nm}$ thick film, which are comparable to those of the commonly used ITO electrode (Supplementary Fig. 2b). Although the AgNW films used in the current work possess a lower transmittance at the blue range due to the plasmonic absorption of the metallic AgNWs, it is reported that this issue can be alleviated by using longer wires with smaller diameters $^{32}$.

To realize fully solution-processed intermediate layers for our tandem devices, we substitute $\mathrm{Ca}$ with $\mathrm{ZnO}$ nanoparticles as the electron-injection material. Taking the green-emitting polymer as an example, an OLED device using $\mathrm{ZnO}$ as the electron injection layer covered with a thermally deposited Ag electrode was fabricated (Figure 2a). As shown in Figure 2b, the $\mathrm{ZnO}$-based cells exhibited similar electroluminescence performance, in terms of the peak brightness and turn-on voltage, to the reference cells based on a Ca interface 
a

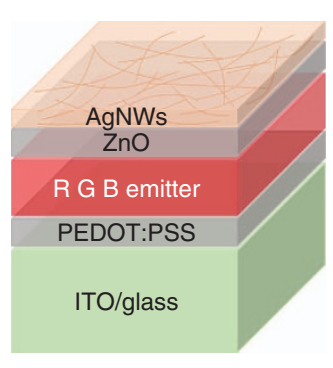

b

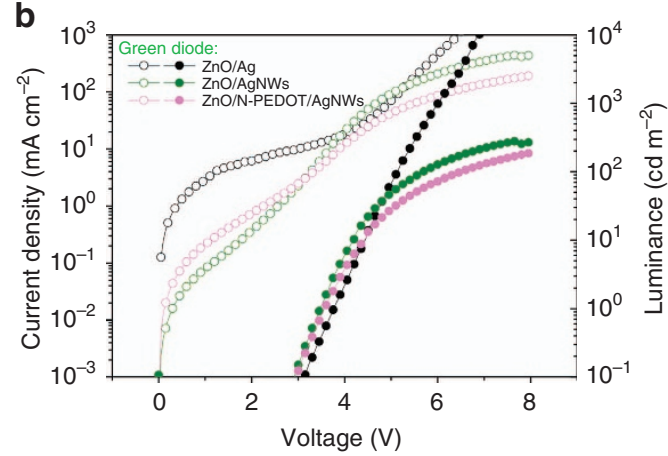

C

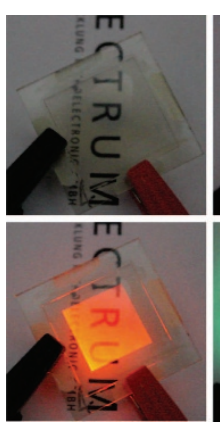

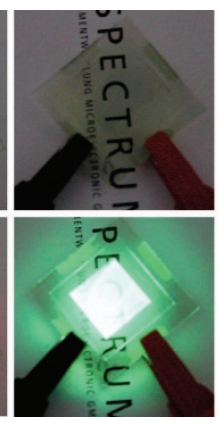

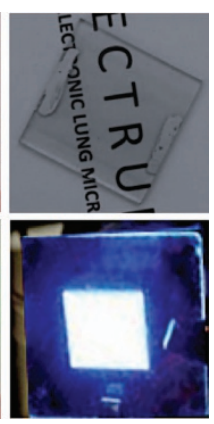

Figure 2 Device structure and electroluminescence performance of semitransparent OLED cells with AgNWs as the cathode. (a) Schematic architecture of semitransparent OLED devices. (b) J-V-L curves of the green-emitting OLED devices with different top electrodes. (c) Photos of the prepared semitransparent red-, green- and blue-emitting cells in their non-emitting (top) and emitting states (bottom).

layer. Similar results were also obtained for the red- and blue-emitting devices, which are presented in Supplementary Fig. 3. These results suggest that the solution-deposited $\mathrm{ZnO}$ nanoparticles are capable of replacing $\mathrm{Ca}$ for constructing efficient OLED devices.

To verify the viability of AgNWs being used as the top electrode to replace the thermally deposited Ag, we further prepared semitransparent OLED devices. It can be seen from Figure $2 \mathrm{~b}$ that the semitransparent green OLED with the AgNW top electrode showed much lower brightness than did its opaque counterparts, which can be explained by the measurement setup used not being able to detect the total emission from both directions. Nevertheless, the semitransparent devices exhibited similar turn-on voltages of $3 \mathrm{~V}$ (green), $2 \mathrm{~V}$ (red, Supplementary Fig. 3a) and $3.5 \mathrm{~V}$ (blue, Supplementary Fig. 3b), implying that the electrons and holes can be efficiently injected from the AgNW network into the device without resistance losses. Photographs of the prepared semitransparent OLED cells are displayed in Figure $2 \mathrm{c}$, where one can see that the devices are fairly transparent in the non-emitting state and show bright emissions under operation. The UV-vis transmission spectra of the three semitransparent devices in their non-emitting state are shown in Supplementary Fig. 4.

We intentionally inserted a thin layer of neutral PEDOT:PSS (denoted as N-PEDOT) between the $\mathrm{ZnO}$ and AgNW layers. The introduced N-PEDOT serves two functions: first, the soft polymer $\mathrm{N}$-PEDOT enables the solution-coated AgNWs to partially embed into the polymer base, which can reduce the roughness of the NW layer, thereby decreasing the chances of shunting. More importantly, it is known that $\mathrm{ZnO}$ can easily be dissolved in acid solvent, and the introduced N-PEDOT prevents the ZnO layer from being dissolved during the deposition of the upper acid PEDOT:PSS 4083 layer in the course of the tandem OLED device fabrication. It is worth noting that although PEDOT:PSS is a widely used hole transport layer with a high work function of $5.2 \mathrm{eV}$, the formation of a $\mathrm{p}-\mathrm{n}$ junction when contacting with $\mathrm{ZnO}$ functions as a charge generation layer that does not negatively affect the electron injection from the AgNWs to the emitting layer ${ }^{33,34}$. In addition, as shown in Supplementary Fig. 5, the symmetric nature of the J-V with the layer sequence of 'ITO/ $\mathrm{ZnO} /$ Neutral PEDOT:PSS/AgNWs' indicates that ohmic contacts have formed at these interfaces, which allow efficient carrier transport between them with minimal losses.

\section{Fabrication of color-tunable tandem OLED cells}

Figure $3 \mathrm{a}$ shows the schematic architecture of the designed colortunable tandem OLED devices. The intermediate AgNW layer was shared by the two subdiodes from which electrons and holes can be injected into the bottom and top subdiodes, respectively. In this 10-layer structure device, apart from the bottom ITO and top Ca/Ag, all of the layers are sequentially deposited from solution by doctor blade coating. AFM was used to investigate the surface properties of the interlayer stack, and the results are shown in Figure 3b. It is found that the front emitting layer deposited on top of the PEDOT:PSS/ITO has a low root-mean-square (RMS) roughness of $1.6 \mathrm{~nm}$ and that the addition of the middle AgNW layer increased the RMS roughness to $13.4 \mathrm{~nm}$. The surface of the AgNWs was then planarized by the covering PEDOT:PSS layer, which reduced the roughness to $9.9 \mathrm{~nm}$ for the back emitting layer. This planarization effect can be best visualized in Figure $3 \mathrm{c}$ by a cross-sectional SEM image. The AgNW meshes are embedded inside the PEDOT:PSS layer, which substantially reduces the roughness of this structure. Furthermore, all of the layers can be identified without obvious intermixing, which is similar to our previous work with a similar interlayer structure ${ }^{35}$. These results indicate a robust stacked layer structure that ensures proper and efficient operation despite the relatively complex device structure.

During the optimization of our tandem devices, we observed that the front subdiode generally exhibits a much lower luminance performance, even when the same emitting material is used for the two subdiodes. An example is shown in Supplementary Fig. 6, where the green emitter was used as both the back and front subdiodes. The front diode exhibited a luminance intensity of $350 \mathrm{~cd} \mathrm{~m}^{-2}$ at an $8 \mathrm{~V}$ bias, which is much lower than that of the back diode of $3739 \mathrm{~cd} \mathrm{~m}^{-2}$. This can be attributed to the light absorption by the back subdiode, where a smaller portion of the light in the front diode is reflected by the back electrode compared to that in the single-junction reference cell where the emitting layer is close to the back reflector. The weaker charge injection of the front diode indicated by the slightly different turn-on voltages of the subdiodes can also contribute to the lower luminance intensity of the front subdiode. From a practical application point of view, it is known that an important feature of a color-tunable display is a balanced emission brightness between the primary colors. It is therefore not trivial to arrange the sequence of the two subdiodes in their tandem configuration. Considering that the human eye has the highest sensitivity toward the green color and the emission in our red OLED shows a deep red emission almost to the edge of the human eye's sensitivity spectrum, we place the green emission material as the front diode and the red-emitting polymer as the back diode.

Figures $3 \mathrm{~d}-3 \mathrm{f}$ shows the J-V-L characteristics of the three colortunable OLED devices with material combinations of green-blue, blue-red and green-red, respectively. When the blue emitter was used as the back diode in combination with the green emitter as the front 
a

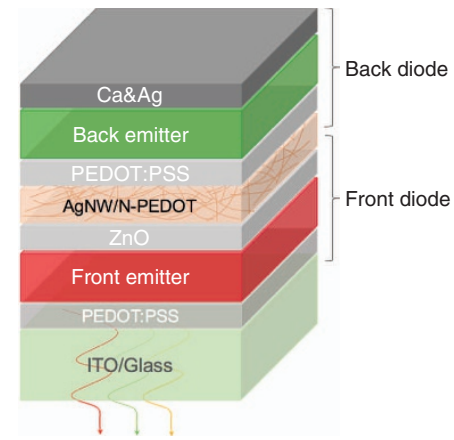

b

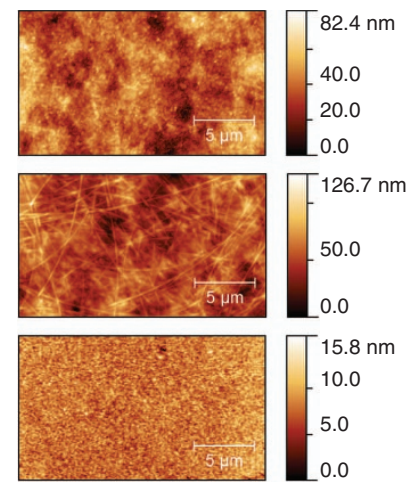

C

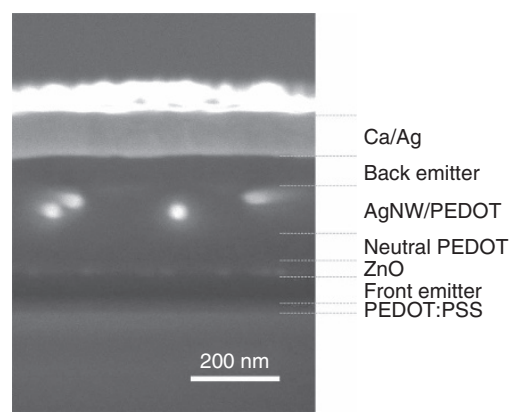

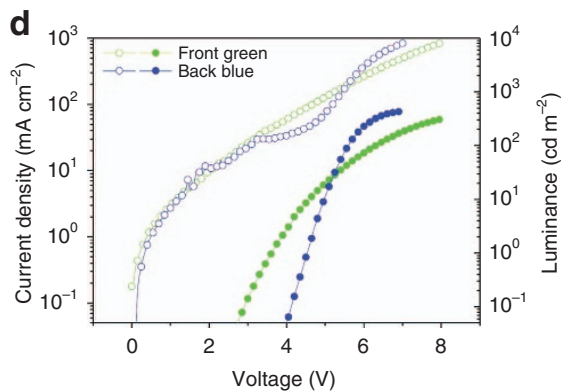
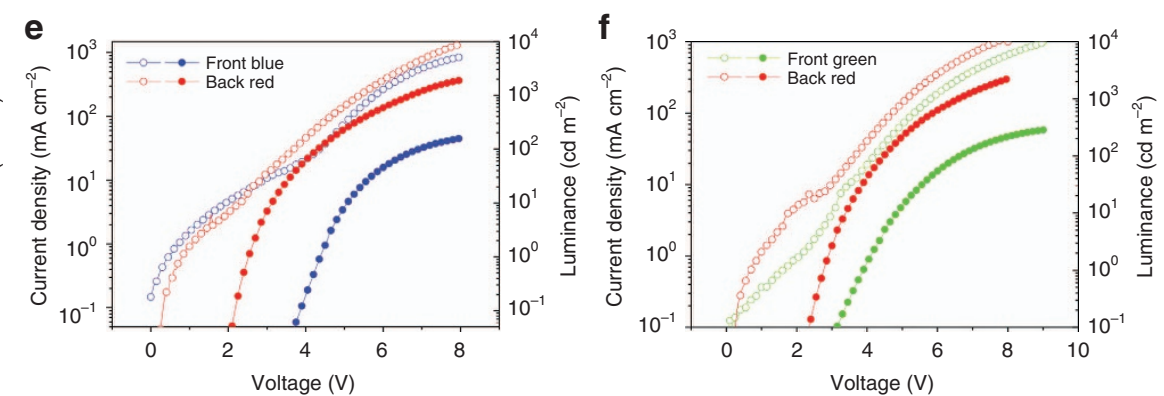

Figure 3 Device architecture, microstructure and electroluminescence performance of the three color-tunable OLED devices with material combinations of green-blue, blue-red and green-red emission polymers. (a) Schematic architecture of the color-tunable OLED device. (b) AFM images of a tandem device until the layers of 'front emitter' (bottom), 'AgNW' (middle) and 'back emitter' (top). Scale bar $=5 \mu \mathrm{m}$. (c) Cross-sectional SEM image of the tandem device. Scale bar $=200 \mathrm{~nm}$. (d-f) J-V-L characteristics of tandem OLED cells with material combinations of green-blue $\mathbf{d}$, blue-red $\mathbf{e}$ and green-red $\mathbf{f}$.

diode, a balanced maximum brightness can be obtained, with $301 \mathrm{~cd} \mathrm{~m}^{-2}$ (at $8 \mathrm{~V}$ ) and $431 \mathrm{~cd} \mathrm{~m}^{-2}$ (at $7 \mathrm{~V}$ ) for the green and blue subdiodes, respectively. For the blue-red and green-red tandem devices, even though the front blue and green subdiodes showed brightness values of 159 and $286 \mathrm{~cd} \mathrm{~m}^{-2}$, respectively, which are much lower than those of the back red-emitting subdiode $\left(2000 \mathrm{~cd} \mathrm{~cm}^{-2}\right)$, a brightness of $100 \mathrm{~cd} \mathrm{~m}^{-2}$ is considered to be sufficient for the display applications. More importantly, all of the subdiodes in these tandem devices exhibited similar turn-on voltages to their reference cells, suggesting that the fully printed intermediate layers have a negligible effect on the optoelectronic performance of the individual diodes. We note that under the current demonstration driving scheme, it would be difficult to realize full white with precise gray levels due to the unbalanced EL intensities, different turn-on voltages and current efficiencies of the two sub-diodes. Additional driving and monitoring circuits will be required when such a device structure is implemented in display systems.

Having established robust intermediate layers for manufacturing tandem OLED devices, we now move to evaluating the colortunability characteristic of these devices. In our tandem OLED device, the middle AgNW layer serves as the electron injection contact for the front diode and the hole injection contact for the back diode. By electrically connecting the top Ag electrode with bottom ITO as one terminal and the middle AgNW layer as the second one, two-terminal tandem devices can be established. These two-terminal devices allow us to selectively manipulate the individual subdiodes by changing the bias direction or applying an alternating current (AC) signal. When a low-frequency square-shaped AC signal with positive and negative potentials is applied to the tandem cells, the two subdiodes can alternatingly turn on and emit pulses of the two primary colors
(Supplementary Movie 1). With a sufficiently high-frequency AC signal applied, mixed colors will be perceived because human eyes are unable to resolve the respective colors emitted by the two subdiodes. Furthermore, by tuning the pulse offset between the positive and negative voltages of an AC signal, the emission intensities of the two colors can be adjusted; thus, a limitless number of colors between the two primary colors can be realized. Supplementary Movie 2 shows the color evolution of the green-red tandem device from red to yellow and ending with green by changing the pulse offset from positive to negative.

Figure $4 \mathrm{a}-4 \mathrm{c}$ presents the measured EL spectra of the three colortunable devices operated by applying AC signals with different magnitude pulse offsets. A square AC signal with a frequency of $100 \mathrm{~Hz}$ and pulse height of $4 \mathrm{~V}$ (equal in both the positive and negative half-cycles) was used to drive the two-terminal tandem OLED devices. In all three devices with tandem combinations of green-blue (Figure 4a), blue-red (Figure 4b) and green-red OLEDs (Figure 4c), when a sufficient offset pulse is applied in either the positive or negative directions, a pure EL spectrum from one of the subdiodes is obtained, as only one of the two subdiodes is turned on. By adjusting the potential offset from positive to negative or vice versa, an intermediate EL spectrum consisting of the combined ELs of the two subdiodes was observed. Figure $4 \mathrm{~d}$ presents the corresponding CIE coordinates of the color-tunable tandem devices calculated from the emission spectra shown in Figure $4 a-4 c$. For all three material combinations, the CIE coordinates are positioned along straight lines between the emission colors of the respective subdiodes, which indicate that all of the colors between the primary colors can be facilely obtained by applying appropriate pulse offsets. No optical micro-cavity effect can be observed in our tandem devices 

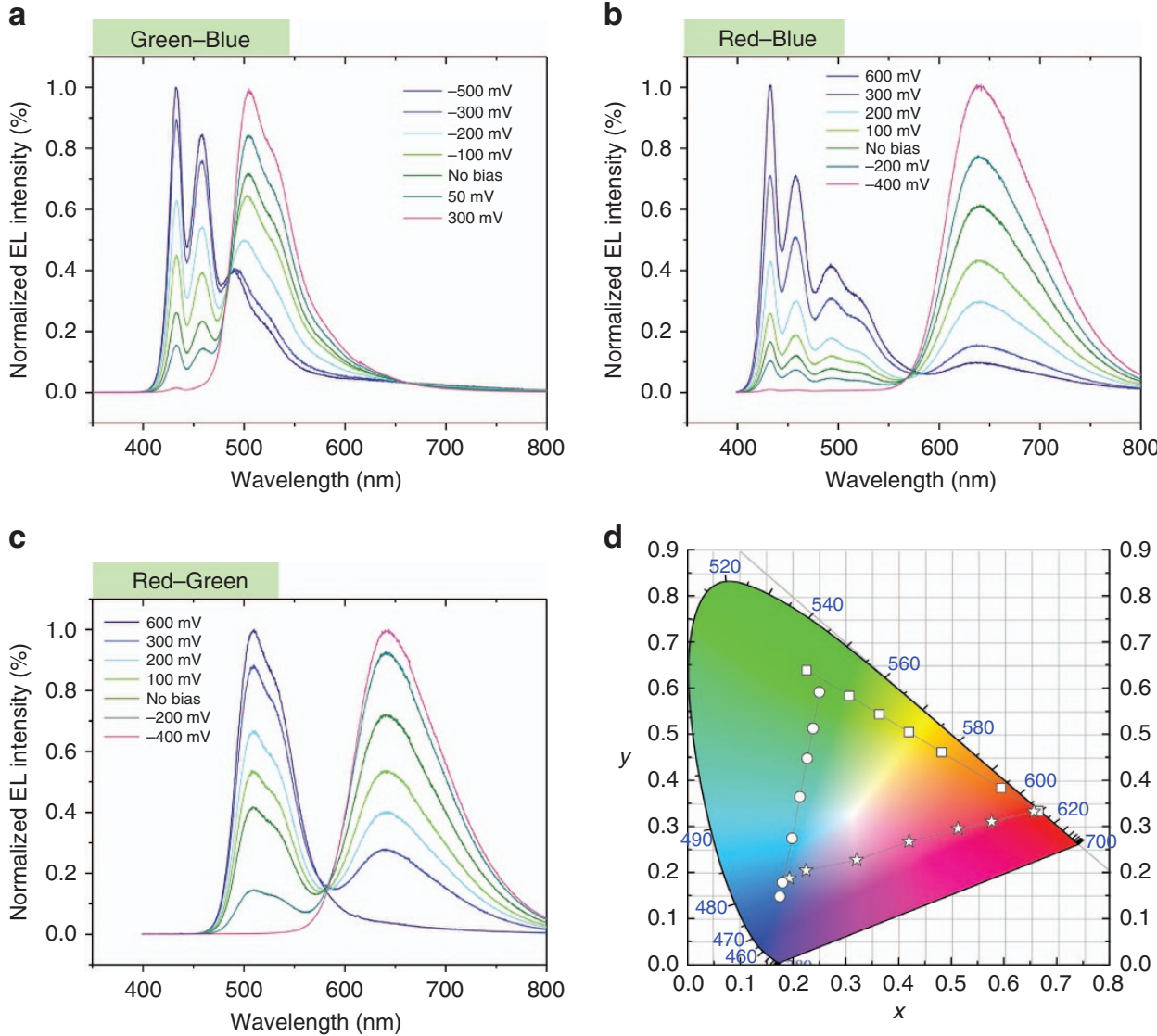

Figure 4 Electroluminescence spectra and the CIE 1931 color space chromaticity coordinates of the three color-tunable OLED devices. (a-c) EL spectrum of the tandem OLED cells with material combinations of green-blue $\mathbf{a}$, red-blue $\mathbf{b}$ and red-green $\mathbf{c}$ operated by applying an $\mathrm{AC}$ signal (100 $\mathrm{Hz}$ ) with different pulse offsets. (d) CIE coordinates of the three color-tunable devices calculated from their EL spectra in a-c.

a

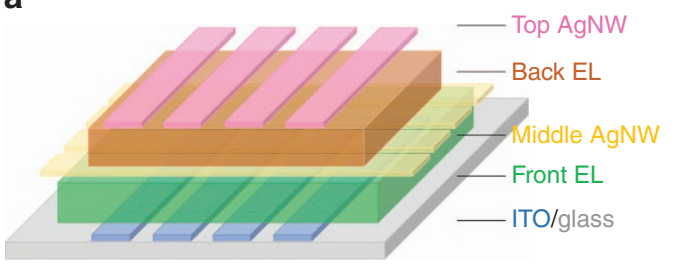

C

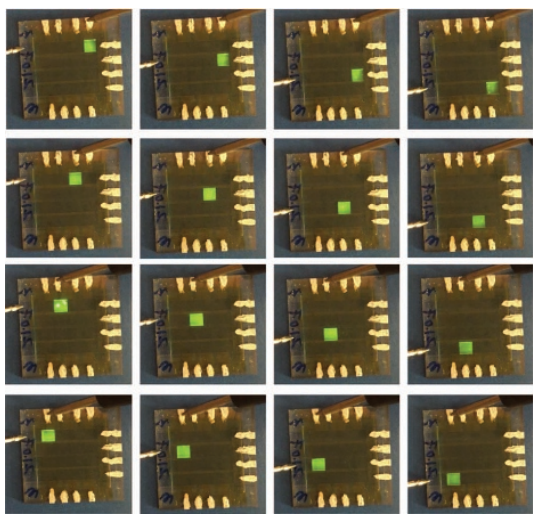

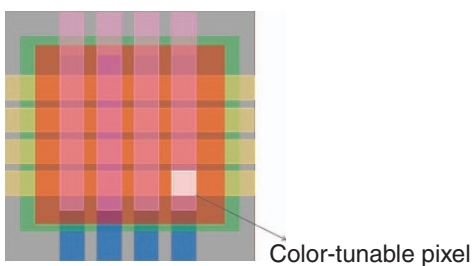

d

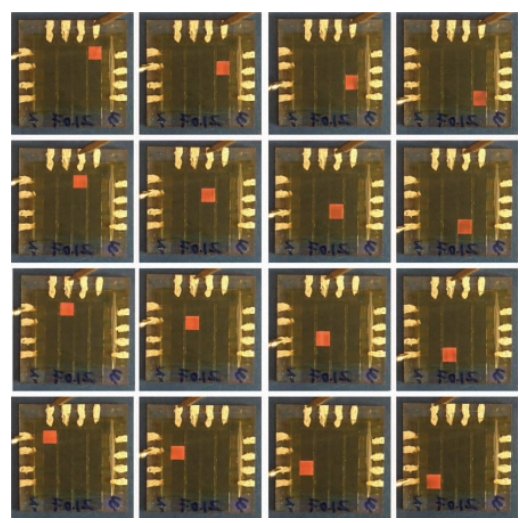

Figure 5 Demonstration of solution-processed color-tunable display. (a) Schematic architecture and (b) top view of the display consisting of 4-by-4 array pixels. In the display, the bottom ITO-coated glass substrate was pre-patterned with a laser, and the middle and top AgNW electrodes were deposited via a modified doctor blade coating. The intersection areas of the bottom, middle and top electrodes define the individual pixels of the display, with each pixel having a size of $2 \times 2 \mathrm{~mm}^{2}$. (c, d) Operation of the individual front green pixels $\mathbf{c}$ and back red pixels $\mathbf{d}$. All the subpixels can be addressed without crosstalk. The continuous operation of all the subpixels is displayed in Supplementary Movie 3. 
(Supplementary Fig. 7), which can be attributed to the use of highly transparent and non-reflective AgNWs as the intermediate electrode.

\section{Manufacture of color-tunable display}

To illustrate a potential application of our color-tunable devices, we designed and fabricated a 4-by-4 pixel matrix display using the material combination of green-red. The procedure for display fabrication is similar to that of the tandem devices described with additional electrode patterning steps. As illustrated in Figure 5a and $5 \mathrm{~b}$, the three charge-injecting electrodes of the device are patterned in four stripes, and the intersections of the three electrodes define individual pixels. We note that the key to the successful manufacture of a display is to construct well-defined electrode stripes with smooth edges, which is of critical importance to avoid crosstalk between neighboring pixels. Transfer printing and lithographic processing have been employed by several groups to fabricate passive matrix displays ${ }^{4,5,7}$. However, additional transfer and lithography steps increase the complexity of the display fabrication, and the reproducibility presents an issue.

For our color-tunable tandem devices, we developed a stripe coating method that allows a much simplified device fabrication procedure. As illustrated in Supplementary Fig. 8, stripe-shaped electrodes with a well-defined area can be facilely solution-deposited using a modifying doctor blade, which enables us to fabricate the 4-by-4 pixel display using a similar procedure to that used for the tandem devices. The step-by-step fabrication of the display is presented in Supplementary Fig. 9. Figure $5 \mathrm{c}$ and $5 \mathrm{~d}$ shows the operation of the prepared colortunable display, demonstrating that all 16 individual front green and back red subpixels can be addressed without crosstalk. The dynamic operation of the display is presented in Supplementary Movie 3. It should be noted that the current demonstration driving scheme may not lead to full color modulation in the OLED display due to the complex timing and offset voltage driving scheme necessary to implement such a design on a large scale. A forward-bias DC signal should be used to drive each individual subdiode independently as a three-terminal device when this structure is implemented in such an application.

\section{CONCLUSION}

In summary, we have demonstrated a technological advance for the fabrication of color-tunable OLED devices by continuous solution deposition. We introduced a highly transparent AgNW layer as an intermediate charge injection contact for the two subdiodes. The accessibility of the AgNW electrode allows the seamless control of the emission spectrum of the tandem device, thereby achieving color tunability. A potential application of our color-tunable tandem devices was illustrated by the fabrication of a 4 -by- 4 pixelated display, which is the first demonstration of a color-tunable display. Considering that the highly efficient intermediate charge injection layers can be facilely prepared by solution processing, we anticipate that full-color OLED devices and displays can be manufactured using such an elegant approach. Overall, we believe that the technology presented in this work will impact the OLED communities by encouraging the development of strategies for the cost-effective manufacture of largearea full-color displays and lighting elements.

\section{CONFLICT OF INTEREST}

The authors declare no conflict of interest.

\section{ACKNOWLEDGEMENTS}

The work was supported by the Cluster of Excellence 'Engineering of Advanced Materials' (EAM) at the University of Erlangen-Nuremberg. FG and KF acknowledge the support of the EU-project SOLPROCEL ('Solution processed high performance transparent organic photovoltaic cells', Grant No. 604506). SC and HC gratefully acknowledge the financial support from the China Scholarship Council (CSC). QX acknowledges the financial support from the South China University of Technology and Deutscher Akademischer Austausch Dienst (DAAD). KF and CJB gratefully acknowledge the financial support through the 'Aufbruch Bayern' initiative of the state of Bavaria.

1 Tang CW, Vanslyke SA. Organic electroluminescent diodes. Appl Phys Lett 1987; 51: 913-915.

2 Friend RH, Gymer RW, Holmes AB, Burroughes JH, Marks RN et al. Electroluminescence in conjugated polymers. Nature 1999; 397: 121-128.

3 Walzer K, Maennig B, Pfeiffer M, Leo K. Highly efficient organic devices based on electrically doped transport layers. Chem Rev 2007; 107: 1233-1271.

4 Liang JJ, Li L, Niu XF, Yu ZB, Pei QB. Elastomeric polymer light-emitting devices and displays. Nat Photonics 2013; 7: 817-824.

5 Müller CD, Falcou A, Reckefuss N, Rojahn M, Wiederhirn V et al. Multi-colour organic light-emitting displays by solution processing. Nature 2003; 421: 829-833.

6 Zheng H, Zheng YN, Liu NL, Ai N, Wang Q et al. All-solution processed polymer lightemitting diode displays. Nat Commun 2013; 4: 1971.

7 Gather MC, Köhnen A, Falcou A, Becker H, Meerholz K. Solution-processed full-color polymer organic light-emitting diode displays fabricated by direct photolithography. Adv Funct Mater 2007; 17: 191-200.

8 Burrows PE, Shen Z, Bulovic V, McCarty DM, Forrest SR et al. Relationship between electroluminescence and current transport in organic heterojunction light-emitting devices. J App/ Phys 1996; 79: 7991-8006.

9 Gu G, Bulović V, Burrows PE, Forrest SR, Thompson ME. Transparent organic light emitting devices. Appl Phys Lett 1996; 68: 2606-2608.

10 Kumar L, Dhawan SK, Kamalasanan MN, Chandra S. Bright-orange organic light emitting diodes fabricated using benzene-naphthalene co-polymer. Thin Solid Films 2003; 441: 243-247.

$11 \mathrm{Gu}$ G, Shen ZL, Burrows PE, Forrest SR. Transparent flexible organic light-emitting devices. Adv Mater 1997; 9: 725-728.

12 Gu G, Parthasarathy G, Burrows PE, Tian P, Hill IG et al. Transparent stacked organic light emitting devices. I. Design principles and transparent compound electrodes. J Appl Phys 1999; 86: 4067-4075.

13 Gu G, Parthasarathy G, Tian P, Burrows PE, Forrest SR. Transparent stacked organic light emitting devices. II. Device performance and applications to displays. J App/ Phys 1999; 86: 4076-4084.

14 Burrows PE, Forrest SR, Sibley SP, Thompson ME. Color-tunable organic light-emitting devices. Appl Phys Lett 1996; 69: 2959-2961.

15 Shen ZL, Burrows PE, Bulović V, Forrest SR, Thompson ME. Three-color, tunable, organic light-emitting devices. Science 1997; 276: 2009-2011.

16 Oliva J, Papadimitratos A, Desirena H, De la Rosa E, Zakhidov AA. Tunable color parallel tandem organic light emitting devices with carbon nanotube and metallic sheet interlayers. J App/ Phys 2015; 118: 194502.

17 Liang CJ, Choy WCH. Tunable full-color emission of two-unit stacked organic light emitting diodes with dual-metal intermediate electrode. J Organomet Chem 2009; 694: 2712-2716.

18 Jiang YB, Lian JR, Chen SM, Kwok HS. Fabrication of color tunable organic lightemitting diodes by an alignment free mask patterning method. Org Electron 2013; 14: 2001-2006.

19 Fröbel M, Schwab T, Kliem M, Hofmann S, Leo K et al. Get it white: color-tunable AC/DC OLEDs. Light Sci Appl 2015; 4: e247.

20 Becker H, Burns SE, Tessler N, Friend RH. Role of optical properties of metallic mirrors in microcavity structures. J Appl Phys 1997; 81: 2825-2829.

21 Zhao YB, Chen R, Gao Y, Leck KS, Yang XY et al. AC-driven, color- and brightnesstunable organic light-emitting diodes constructed from an electron only device. Org Electron 2013; 14: 3195-3200.

22 Cho SH, Kim EH, Jeong B, Lee JH, Song G et al. Solution-processed electron-only tandem polymer light-emitting diodes for broad wavelength light emission. $J$ Mater Chem C 2017; 5: 110-117.

23 Fung MK, Li YQ, Liao LS. Tandem organic light-emitting diodes. Adv Mater 2016; 28 : 10381-10408.

24 Höfle S, Schienle A, Bernhard C, Bruns M, Lemmer U et al. Solution processed, white emitting tandem organic light-emitting diodes with inverted device architecture. Adv Mater 2014; 26: 5155-5159.

$25 \mathrm{Pu}$ YJ, Chiba T, Ideta K, Takahashi S, Aizawa N et al. Fabrication of organic lightemitting devices comprising stacked light-emitting units by solution-based processes. Adv Mater 2015; 27: 1327-1332.

26 Höfle S, Bernhard C, Bruns M, Kübel C, Scherer T et al. Charge generation layers for solution processed tandem organic light emitting diodes with regular device architecture. ACS Appl Mater Interfaces 2015; 7: 8132-8137. 
27 Chiba T, Pu YJ, Kido J. Solution-processed white phosphorescent tandem organic lightemitting devices. Adv Mater 2015; 27: 4681-4687.

28 Lee JY, Connor ST, Cui Y, Peumans P. Solution-processed metal nanowire mesh transparent electrodes. Nano Lett 2008; 8: 689-692.

29 Langley D, Giusti G, Mayousse C, Celle C, Bellet D et al. Flexible transparent conductive materials based on silver nanowire networks: a review. Nanotechnology 2013; 24: 452001.

30 Guo F, Zhu XD, Forberich K, Krantz J, Stubhan T et al. ITO-free and fully solutionprocessed semitransparent organic solar cells with high fill factors. Adv Energy Mater 2013; 3: 1062-1067.

31 Li L, Yu ZB, Hu WL, Chang CH, Chen Q et al. Efficient flexible phosphorescent polymer light-emitting diodes based on silver nanowire-polymer composite electrode. Adv Mater 2011; 23: 5563-5567.

32 Lee JH, Lee P, Lee D, Lee SS, Ko SH. Large-scale synthesis and characterization of very long silver nanowires via successive multistep growth. Cryst Growth Des 2012; 12 5598-5605.

33 Lee JH, Kim JW, Kim SY, Yoo SJ, Lee JH et al. An organic $p-n$ junction as an efficient and cathode independent electron injection layer for flexible inverted organic light emitting diodes. Org Electron 2012; 13: 545-549.
34 Chen YH, Ma DG, Sun HD, Chen JS, Guo QX et al. Organic semiconductor heterojunctions: electrode-independent charge injectors for high-performance organic light-emitting diodes. Light Sci Appl 2016; 5: e16042.

35 Guo F, Li N, Fecher FW, Gasparini N, Quiroz COR et al. A generic concept to overcome bandgap limitations for designing highly efficient multi-junction photovoltaic cells. Nat Commun 2015; 6: 7730.

cc)(1) (2) This work is licensed under a Creative Commons Attribution-

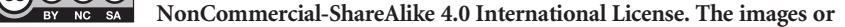
other third party material in this article are included in the article's Creative Commons license, unless indicated otherwise in the credit line; if the material is not included under the Creative Commons license, users will need to obtain permission from the license holder to reproduce the material. To view a copy of this license, visit http:// creativecommons.org/licenses/by-nc-sa/4.0/

(C) The Author(s) 2017

Supplementary Information for this article can be found on the Light: Science \& Applications' website (http://www.nature.com/sa). 